\title{
Rapidly Ongoing Acroosteolysis in Rheumatoid Arthritis: A Case Report
}

\author{
Habibi Leila*, Imane El Bouchti
}

Department of Rheumatology, Mohammed VI University Hospital, Marrakech, Morocco

DOI: $10.36347 /$ sjmcr.2021.v09i02.006

| Received: 23.01.2021 | Accepted: 08.02.2021 | Published: 11.02.2021

*Corresponding author: Habibi Leila

Abstract

Case Report

Acroosteolysis refers to a destructive process involving parts of the distal phalynx of fingers or toes that may extend to metacarpals or metatarsals. Rarely primary or idiopathic, it is often acquired and requires an etiological investigation. Rheumatoid arthritis (RA) is an exceptional etiology of acroosteolysis occuring as a result of rheumatoid vasculitis, peripheral neuropathy or the extension of distal interphalangeal joint damage. We report the case of a 50-year-old female patient with history of diabetes mellitus and seropositive and erosive RA evolving for 8 years, currently active. The recent radiological assessment showed osteolysis of the distal phalanges of both hands, mainly the left one, with a negative etiological investigation, therefore reviving the debate on the Acroosteolysis-Rheumatoid arthritis association.

Keywords: Acroosteolysis, Rheumatoid Arthritis.

Copyright $\left({ }^{\circ} 2021\right.$ The Author(s): This is an open-access article distributed under the terms of the Creative Commons Attribution 4.0 International License (CC BY-NC 4.0) which permits unrestricted use, distribution, and reproduction in any medium for non-commercial use provided the original author and source are credited.

\section{INTRODUCTION}

Acroosteolysis is a rare condition. The diagnosis of primary acroosteolysis can only be made after eliminating other causes, especially rheumatological, toxic and micro traumatic [1]. We report the case of a patient followed in the department of rheumatology for Rheumatoid arthritis (RA) with recent radiological acroosteolysis affecting the distal phalanges of both hands.

\section{Case Report}

Mrs. $\mathrm{H} \mathrm{M}$ is a 50-year-old patient, with medical history of type 2 diabetes mellitus controlled with diet and medical therapy, non-alcoholic steatohepatitis, and RA evolving for 8 years for which she received methotrexate $20 \mathrm{mg}$ per week combined with $10 \mathrm{mg}$ per day of prednison with persistence of the disease activity.

On examination, the patient had poly synovitis involving metacarpophalangeal and proximal interphalangeal joints of the fingers. The squeeze test was positive in both hands. She had stiffness of both wrists, and gooseneck-like deformities of the fingers. Laboratory tests showed an inflammatory syndrome with accelerated erythrocyte sedimentation rate at 46 $\mathrm{mm} /$ hour and $\mathrm{C}$ reactive protein at $57 \mathrm{mg} / 1$, normal complete blood count and serum protein electrophoresis revealed elevation of alpha 2 and beta 2 . The immunological assessment was positive with anticyclic citrullinated peptide (anti-CCP) antibodies > $200 \mathrm{IU} / 1$ $(\mathrm{N}<25)$, a rheumatoid factor at $128(\mathrm{~N}<15)$. The antinuclear antibodies were negative.

X-rays from the previous year had shown structural damage to both carpal, metacarpophalangeal, proximal and distal interphalangeal joints without demineralization or acroosteolysis (Figure-1). Current radiological assessment showed, in addition to the previous lesions, the appearance of resorption of the phalangeal tufts of both hands with subperiosteal resorption of the second phalanges of the hands (Figure2).

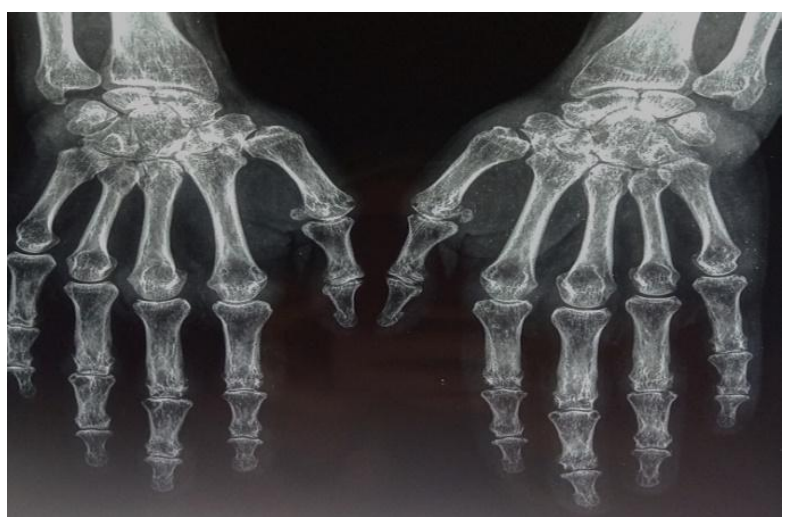

Fig-1: X-ray of both hands facing from the previous year showing geodes and erosions of both carpals, MCP, PPI and IPD associated with bilateral radiocarpal pinching 
Habibi Leila \& Imane El Bouchti., Sch J Med Case Rep, Feb, 2021; 9(2): 132-134

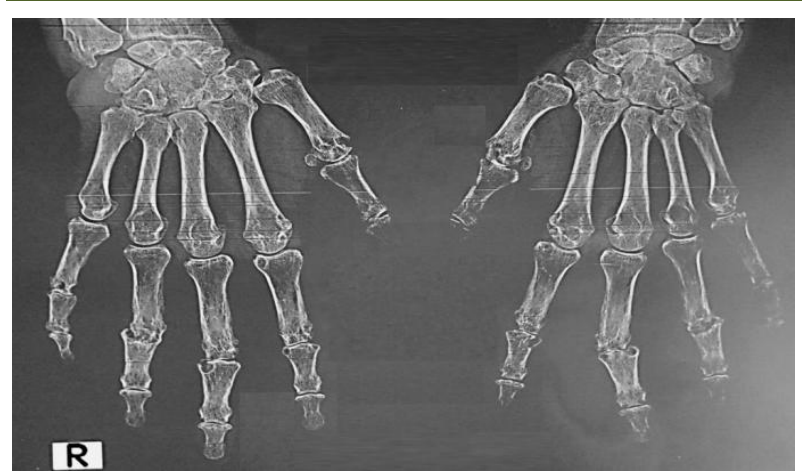

Fig-2: X-ray of both hands facing, from the recent hospitalization. Structural progression of both carps, MCP, PPI, IPD and onset resorption of the phalangeal tufts of both hands marked on the left with a subperiosteal resorption of the second phalanges of the left hand

The medical history didn't show any personal or family psoriasis, frostbite of the hands or Raynaud's phenomenon. The patient had no dry eye syndrome and no respiratory signs. Also, no toxic exposure, particularly to vinyl chloride was found.

In addition, physical examination did not find any signs pointing to a secondary cause of acroosteolysis, particularly sclerodactyly, trophic disorders of the fingers, rashes, nodules or skin ulcerations next to the last phalanges, skin psoriasis, or facial dysmorphia. Peripheral pulses were present and symmetrical. Neurological evaluation showed no abnormalities. Examination of the spine, sacroiliacs and entheses was normal.

Furthermore, paraclinical examinations were performed including the electromyogram of the four limbs which did not show peripheral neuropathy. Magnetic resonance imaging of the sacroiliacs showed degenerative sacroiliitis. The $\mathrm{x}$-ray of the hands and forefeet showed no subcutaneous calcinosis. The chest CT was normal. The accessory salivary gland biopsy did not show any granuloma or signs of amyloidosis. The converting enzyme was normal. Phosphocalcic assessment was normal including PTH1-84. Blood sugar was 1.15 and glycated hemoglobin was normal at $6.3 \%$. Blood tests were negative for hepatitis B and C, syphilis and the acquired immunodeficiency virus infection. On the other hand, proteinuria was positive at $530 \mathrm{mg} / 24 \mathrm{~h}$, with microscopic hematuria. Renal biopsy showed normal renal parenchyma with 3 sclerotic glomeruli. Ophthalmologic examination didn't point out any signs of diabetic retinopathy.

The patient received tocilizumab at a dose of 8 $\mathrm{mg} / \mathrm{kg} / 4$ weeks with good progress and achievement of remission within the first infusion. The current follow-up is 6 months.

\section{DiscusSiON}

Acroosteolysis can be primary, idiopathic, or acquired. Acquired acroosteolysis is by far the most frequent and must be eliminated first before considering an idiopathic cause [1].

It includes various etiologies such as primary or secondary hyperparathyroidism which has the particularity of associating a subperiosteal resorption of the radial edge of the 2 nd phalanx with acroosteolysis on standard radiography $[1,2]$ and the diagnosis is based on carrying out the phosphocalcic assessment.

Rheumatological causes include psoriatic arthritis, which combines acroosteolysis with distal interphalangeal joint damage [3, 4], multicentric reticulohistiocytosis, which describes a characteristic vesicular skin lesions, erosive arthritis and acroosteolysis [1], and scleroderma, which includes sclerodactyly lesions, trophic skin disorders, Raynaud's disease, subcutaneous calcinosis and distal longitudinal acroosteolysis [5]. Other chronic inflammatory rheumatisms that may be accompanied by acroosteolysis are described in case reports, such as systemic lupus, mixed connectivitis and sarcoidosis [6, 7].

Among the secondary etiologies, we also find neuroarthropathies secondary to diabetes, syringomyelia, tabes, leprosy and acropathy ulceromutilant [3]. Diagnosis is based on interrogation, neurological examination and biological workup including syphilitic serology, Electromyogram data, and sometimes skin biopsy.

Acroosteolysis is also described after a burn, frostbite or repetitive strain injuries in guitar players for example [1,7]. It is then localized within the radius adjacent to the skin involvement.

Acroosteolysis can also be caused by vinyl chloride poisoning [3, 7, 8]. The primary forms of acroosteolysis are dominated by Hadju-Cheney syndrome, an autosomal dominant disorder characterized by short stature, facial dysmorphism, osteoporosis, periodontal disease, and distal acroosteolysis giving an appearance of digital pseudo hippocratism [9]. The other primary forms are Faber's disease, Winchester syndrome, hereditary multicenter carpocentric osteolysis, Joseph and Shinz acroosteolysis and Pycnodysostosis $[7,10]$.

In rare cases, acroosteolysis remains idiopathic, characterized by rapidly extensive osteolysis associated with erosions [10].

Rheumatoid arthritis is exceptionally described as the etiology of acrosteal disease. The possible pathophysiologic data point to the link with rheumatoid vasculitis, associated neuropathy or the extension of distal interphalangeal damages, although rare [3, 11]. Our observation is singular by the presence of acroosteolysis of rapid onset and extension, suggesting after the negativity of the detailed etiological assessment, three diagnostic possibilities: acroosteolysis 
punctuating the development of severe RA, a RA association - psoriatic arthritis in front of the presence of the attack of the distal phalanx, and the signs of periostitis but the patient did not have skin psoriasis, neither familial nor personal, and the standard $\mathrm{x}$-ray of the sacroiliac was normal. The MRI was negative which does not rule out a strictly peripheral form of psoriatic arthritis. Another hypothesis is the succession over time of two different conditions, each evolving on their own account: RA and idiopathic acroosteolysis. Finally, the appearance of acroosteolysis during the last year of the course of RA which was marked by a high activity of the disease with persistent biological inflammatory syndrome raises the possibility of a link between the severe activity of disease and the onset of acroosteolysis. Recent data suggest the involvement of the RANK / RANKL system and pro-inflammatory cytokines in local and general bone demineralization in patients with rheumatoid arthritis. This demineralization can affect the phalangeal tufts and cause acroosteolysis, provided that the rheumatoid arthritis is seropositive and active, which is the case in our patient. Anti-CCP antibodies and interleukins are at the centre of this pathophysiology.

\section{CONCLUSION}

Acroosteolysis in adults is uncommon. It can have various etiologies. It requires detailed etiological investigation in order to rule out a secondary cause. Our singular observation illustrates the possible occurrence of rapidly extensive acroosteolysis in a field of active rheumatoid arthritis.

\section{REFERENCES}

1. Drapé J, Chevrot A, Gires F, Sarazin L, Baran R. Acro-ostéolyse de l'adulte. EMC - Radiologie et imagerie médicale - Musculosquelettique Neurologique - Maxillofaciale. 2003 Jan;1(1):1-6.

2. Bennett J, Suliburk JW, Morón FE. Osseous Manifestations of Primary Hyperparathyroidism: Imaging Findings. International Journal of Endocrinology. 2020 Feb 21;2020:1-10.

3. Kemp SS. Acro-osteolysis. JAMA. 1986 Apr 18;255(15):2058.

4. Mol S, Kraan G. Osteolysis of the terminal phalanges of the hand. BMJ Case Rep. $2016 \mathrm{Apr}$ 26;2016:bcr2016214527

5. Sandler RD, Matucci-Cerinic $M$, Hughes $M$. Musculoskeletal hand involvement in systemic sclerosis. Seminars in Arthritis and Rheumatism. 2020 Apr;50(2):329-34.

6. Davies K, Bukhari M, Ottewell L. Acroosteolysis in a patient with systemic lupus erythematosus/mixed connective tissue disease. Rheumatology (Oxford). 201903 1;58(3):426.

7. Botou A, Bangeas A, Alexiou I, Sakkas LI. Acroosteolysis. Clin Rheumatol. 2017 Jan; 36(1):9-14

8. Magnavita N. Acro-osteolysis caused by vinyl chloride. Med Lav. 2010 Sep-Oct;101(5):395.

9. Rochoux Q, Léon N, Marcelli C. Le syndrome d'Hajdu-Cheney ou acro-ostéolyse autosomique dominante. Revue du Rhumatisme Monographies. 2019 Apr;86(2):132-7

10. Harzy T, Benbouazza K, Lazrak N, Amine B, Hajjaj-Hassouni N. Idiopathic phalangeal acroosteolysis: a case report. Joint Bone Spine. 2003 Apr;70(2):146-8

11. Rohlfing BM, Basch CM, Genant HK. Acroosteolysis as the sole skeletal manifestation of rheumatoid vasculitis. BJR. 1977 Nov; 50(599):830-3. 